\title{
Effect of response-contingent vs. noncontingent shock on ducklings' preference for novel imprinting stimuli
}

\author{
PETER DePAULO, HOWARD S. HOFFMAN, SANDRA KLEIN \\ and STEPHEN GAIONI \\ Bryn Mawr College, Bryn Mawr, Pennsylvania 19010
}

\begin{abstract}
Individual ducklings received electrical shock in the presence of an imprinting stimulus whenever they pecked at food. Other ducklings received an identical series of shocks in the presence of an imprinting stimulus, but for them shock delivery was independent of their pecking behavior. In a subsequent session, the use of shock was discontinued and all birds were afforded the opportunity to approach either the imprinting stimulus (i.e., the stimulus previously present during shock) or a novel imprinting stimulus that was simultaneously presented. Ducklings that were shocked when they pecked at food either exhibited no preference or they preferred the original imprinting stimulus. In contrast, birds for whom shock was independent of their feeding behavior preferred the novel stimulus. These findings imply that the delivery of shock in the presence of an imprinting stimulus can endow the stimulus with conditioned aversive properties. They also imply that the stimulus will acquire little or no aversiveness if shock delivery is contingent upon a specific response such as pecking.
\end{abstract}

When a parental figure punishes or inflicts pain upon its offspring, the attachment behavior of the young organism often continues unabated and may even be intensified (Bowlby, 1969; Harlow \& Harlow, 1962). This phenomenon has a parallel in the imprinting literature. If a duckling is given electric shock while being exposed to a surrogate attachment object (a moving imprinting stimulus), the subject's tendency to approach and follow the object may be enhanced (Kovach \& Hess, 1963). Students of imprinting have found this somewhat puzzling, because it seemed to imply that the approach-eliciting properties of the imprinting object are somehow strengthened by the occurrence of aversive stimulation in its presence. More recently, however, this interpretation of the effect of the shock was called into question by Barrett (1972). In Barrett's study, ducklings were shocked in the presence of an imprinting stimulus and were subsequently allowed to choose between that stimulus and a similar but visually distinctive stimulus that was simultaneously presented. In the first phase of the experiment, the subjects followed the stimulus more closely (thus replicating the findings of Kovach and Hess), but in the choice test these subjects preferred the new stimulus. Since control (not shocked) subjects either showed no preference or

This research was supported by National Institute of Mental Health Grant MH 19715, directed by Howard S. Hoffman. Requests for reprints should be addressed to Howard S. Hoffman, Department of Psychology, Bryn Mawr College, Bryn Mawr, Pennsylvania 19010. Dr. DePaulo is now at St. Joseph's College, Philadelphia, Pennsylvania. Dr. Gaioni is now at Washington University, St. Louis, Missouri. preferred the original stimulus, it is clear that the effect is not consistent with the suggestion that the approach-eliciting properties of an imprinting stimulus are augmented by occurrences of aversive stimulation.

These seemingly paradoxical effects-enhanced following despite a preference for a novel stimulushave implications for a theoretical issue that has been raised about the nature of imprinting itself. According to traditional ethological theory, imprinting cannot be adequately understood in terms of the concepts and principles of associative learning. The enhanced-following effect has been held in support of this view. As Hess (1973) has noted, subjects that are shocked in the presence of a given stimulus would be expected to associate the stimulus with shock, and, as a result, their tendency to approach and follow the stimulus should be reduced. According to this argument, the fact that following behavior is actually augmented by shock implies that some process other than associative learning is involved. On the other hand, the behavior of the subjects in a choice situation is in line with conditioning principles because under these circumstances the subjects do show a reduced tendency to approach a stimulus that was present during shock.

In a recent series of studies, Ratner (1976) replicated both of these seemingly contradictory effects and also examined what happens when the delivery of shock is restricted to periods during which the imprinting stimulus has been briefly withdrawn. He found that this operation, like the delivery of shock in the presence of the stimulus, yielded enhanced 
following behavior when only the original stimulus was available. However, it failed to generate a preference for a novel stimulus when the subject was subsequently allowed a choice.

Ratner interpreted his results as implying that aversive stimulation has two kinds of behavioral effects in the context of imprinting: (a) it increases the subjects' motivation to engage in filial behavior and (b) it results in the conditioning of aversive reactions to stimuli that are present when the aversive events occur. According to Ratner's account, the enhancement of following behavior exhibited by shocked subjects when only the original imprinting stimulus is present is a manifestation of the subjects' increased motivation to engage in filial behavior. Presumably, the subject approaches the imprinting stimulus despite any fear it may arouse because the subject has nowhere else to turn. But if the subject is subsequently offered an alternative stimulus (that is not too different), it prefers the alternative. That the preference for the alternative stimulus is the result of a conditioned fear reaction to the original stimulus is suggested by Ratner's finding that when subjects were shocked only in the absence of the imprinting stimulus they did not subsequently prefer a novel one.

Ratner's account of the effects of aversive stimulation does not assume the operation of any processes unique to imprinting. The enhanced following effect is attributed to motivational properties of the shock, and this kind of behavioral control by aversive stimulation has been noted in situations other than imprinting. For example, aggressive behavior (Azrin, Ulrich, Hutchinson, \& Norman, 1964; O'Kelley \& Steckle, 1939) and sexual behavior (Barfield \& Sachs, 1968; Caggiula \& Eibergen, 1969), can be energized by aversive events. The associative effects of shock as reflected in the preference shift also have counterparts in the literature of learning. For example, animals will refrain from entering goalboxes that have been associated with shock when an appropriate alternative is available. The present work represents a continuation of the analysis of aversive reactions in the context of imprinting via the concepts and principles of learning and motivation. In particular, the research asked whether the preference for the novel, but similar, stimulus could be reduced or eliminated if shock were delivered in the presence of an imprinting stimulus but were made contingent upon the occurrence of a given response rather than being independent of subjects' behavior. This kind of manipulation has been found to attenuate the associative effects of shock in another context. A study by Orme-Johnson and Yarczower (1974) has revealed that when shock is delivered to pigeons in the presence of an exteroceptive stimulus (a key light), that stimulus acquires conditioned aversive properties if the shock is response independent but not if the shock is response contingent. Analogously, it seemed possible that if shock delivered to ducklings in the presence of an imprinting stimulus is made contingent upon a given response (for example, pecking at food), then that stimulus might acquire little or no conditioned aversiveness, and subjects might not subsequently prefer a novel stimulus if offered a choice.

\section{METHOD}

\section{Subjects}

Twelve Khaki Campbell ducklings (Anas platyrhynchos domesticus) were hatched in visual isolation in a forced-air incubator from eggs obtained from George F. Shaw, Inc., West Chester, Pennsylvania. Each duckling was maintained in an individual housing unit consisting of a 56.7-liter translucent plastic container that was lined with a disposable polyethylene bag partially filled with absorbent bedding material (San-i-cel). Water was continually available within each housing unit. Food (dry mash) was also available, except during periods when deprivation conditions were in force.

\section{Apparatus}

Two identical experimental chambers were employed. Each chamber consisted of a plywood box $(140 \times 76 \times 76 \mathrm{~cm})$, divided lengthwise by a fine-mesh stainless steel screen to form two compartments, a stimulus compartment $(140 \times 30 \times 76 \mathrm{~cm})$ and a subject compartment $(140 \times 46 \times 76 \mathrm{~cm})$. Lighting in the subject compartment was provided by two continuously illuminated 75 -W incandescent bulbs mounted above the screen. These bulbs were positioned such that the light reflected from the screen prevented the subject from viewing into the stimulus compartment unless that compartment was also illuminated. The floor area of the subject compartment was covered by black rubber matting and was delineated into four equal-size quadrants $(70 \times 23 \mathrm{~cm})$ by photocell units. Electrical signals produced by interruption of the photocell beams were routed to a system of lock-down relays programmed to record automatically the location of the subject during the experimental procedures.

The imprinting stimulus consisted of a white rectangular foamrubber object $(23 \times 10 \times 10 \mathrm{~cm})$ mounted over a model train engine. Presentations of the stimulus were produced by illuminating two overhead 75-W incandescent bulbs in the stimulus compartment and moving the stimulus back and forth along HO-gauge track at approximately $30 \mathrm{~cm} / \mathrm{sec}$.

The novel stimulus was identical to the imprinting stimulus except that black diagonal stripes of adhesive tape were placed on its white foam-rubber covering.

Electrical shock was generated by an Applegate constant-current stimulator and delivered to the duckling through a system that has been fully described elsewhere (Hoffman \& Ratner, 1974). Each duckling wore a pair of permanently fastened wingbands constructed of fine gold chain. During experimental procedures, the wingbands were connected to a pair of lightweight miniature clamps that were suspended from above the apparatus. With this arrangement, ducklings could freely move about the subject compartment and shock could be delivered at any time.

Experimental events were programmed through conventional relay equipment and documented on an Esterline-Angus event recorder. In addition, the ducklings were observed during the experimental procedures over a closed-circuit television system.

\section{Procedure}

Approximately $16 \mathrm{~h}$ after hatching, each duckling was removed from the incubator, fitted with wingbands, and individually placed in the experimental chamber for a 30 -min imprinting session. During the session, the wingbands were connected to the shock delivery system, but no shocks were delivered. Throughout the 
session, the moving imprinting stimulus was continuously present. Two additional 30 -min imprinting sessions were given to each duckling within $24 \mathrm{~h}$ of the first session. During all sessions, dry mash was scattered along the length of the subject compartment near the screen which separated the subject and stimulus compartments.

On Day 3 posthatch, each duckling was randomly assigned to one of two equal-size groups $(\mathrm{N}=6)$ : contingent shock and noncontingent shock. Six pairs of subjects were formed, with each pair containing a member of each of the two groups.

The two mem'ers of a given pair were deprived of food for approximately $10 \mathrm{~h}$ on Day 3 and were then placed simultaneously into the experimental chambers located in separate soundproof rooms for a $30-\mathrm{min}$ experimental session. Al:hough the two chambers were identical, the assignment of subjects to chambers was counterbalanced so that each chamber was used for the duckling given contingent shock in three of the pairs and for the duckling given noncontingent shock in the other three pairs. Throughout this session, the imprinting stimulus was continuously present and moving along the full length of each apparatus. Food was scattered along the floor of the subject compartment of each chamber as it was during imprinting sessions.

During this session, two experimenters were employed. One experimenter observed the duckling assigned to the contingentshock condition via closed-circuit television, and operated a switch whenever the subject pecked at the food on the floor. Switch closure delivered a 1-mA, 300-msec shock to both members of the pair being run. Thus, shock delivery was contingent on peck responses for the duckling assigned to the contingent-shock group, but was delivered independently of the behavior of the duckling assigned to the noncontingent shock group. Food-peck responses of the duckling given noncontingent shock had no programmed consequences but were recorded by the other experimenter, who operated a separate switch. Three additional 30 -min sessions of this type were given to each pair of ducklings over the next 2 days. The experimenters reversed their assignments between sessions so that each experimenter observed subjects in the noncontingent shock and contingent shock groups equally often. Sessions occurred approximately every $12 \mathrm{~h}$. Prior to each session, the ducklings were deprived of food for $10 \mathrm{~h}$, and immediately following the session they were given 90 min of unlimited access to food in their housing units.

Although following behavior was recorded during the imprinting sessions in the Barrett (1972) and Ratner (1976) studies, no attempt was made to monitor following responses in the present experiment because a competing response (eating) was made available.

On Day 6, each duckling was given a preference test, during which the imprinting stimulus and the novel stimulus were simultaneously presented and withdrawn in 10 cycles. Each cycle consisted of a $60-\mathrm{sec}$ period of stimulus presentation followed by a 30-sec period of stimulus withdrawal. During stimulus presentation periods, both stimuli were visible and continually moved back and forth along one-half the length of the apparatus; that is, one stimulus was restricted to the left half of the track and the other to the right half. To eliminate the effect of possible preferences for the left or right side of the subject compartment, the locations of the imprinting stimulus and the novel stimulus were reversed during the fifth stimulus withdrawal period. During each stimulus presentation period, the times spent by the subject in the quadrant closest to the imprinting stimulus and in the quadrant closest to the novel stimulus were separately recorded.

Food was removed from the floor of the subject compartment prior to the preference test, and although no shocks were administered, the subjects were connected to the shock delivery system.

\section{RESULTS}

Figure 1 shows the mean number of food-peck responses recorded for each group in each of the four

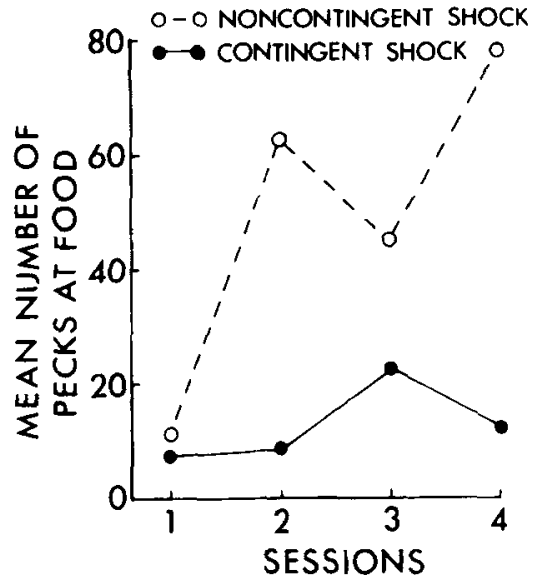

Figure 1. Food-peck responses during imprinting sessions by subjects given shocks contingent upon food pecks and by subjects given response-independent shock.

experimental sessions. (The function for the contingentshock group also represents the mean number of shocks given to subjects in both conditions in each session.) In each session, the contingent-shock group emitted fewer pecks than the noncontingent shock group. A repeated-measures analysis of variance revealed a significant effect of Groups $[F(1,10)=5.29$, $\mathrm{p}<.05]$. Thus, the suppression (punishment) of food-peck responses by contingent shock was statistically reliable. The main effect of Sessions and the interaction of Groups by Sessions were not significant $[\mathrm{Fs}(3,30) \leqslant 1.00$, ps $>.05]$.

Figure 2 shows the mean percent of the total stimulus presentation time that ducklings spent in the quadrants closest to the imprinting and novel stimuli during the preference test. The ducklings exposed to noncontingent shock spent more time in the quadrant closest to the novel stimulus than in the quadrant closest to the original imprinting stimulus. Ducklings for whom shock was response-contingent spent more time in the presence of the original imprinting stimulus. A two-factor mixed-design analysis of variance yielded a significant interaction between the main

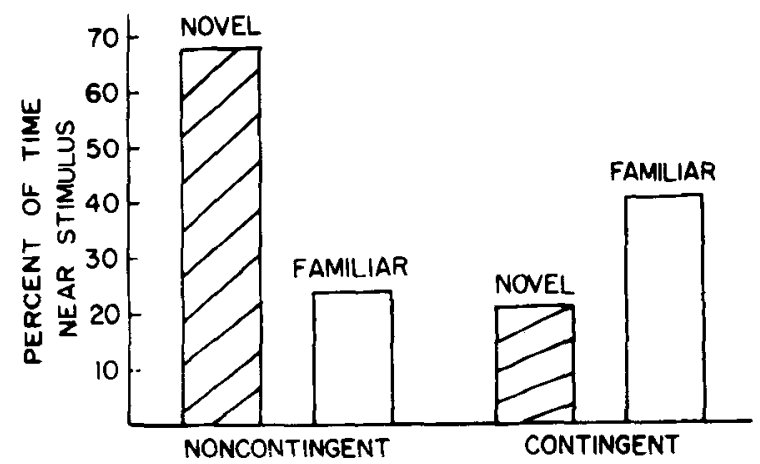

Figure 2. Percent of total stimulus presentation time spent in proximity to the imprinting stimulus associated with shock and to the novel stimulus during the preference test. The legends on the abscissa refer to the experimental groups. 
effects of Groups and Stimuli $[\mathrm{F}(1,10)=7.11, \mathrm{p}<$ $.05]$. Thus, the contingency manipulation reliably altered subjects' choices of the novel vs. familiar stimuli. The main effects of Groups and Stimuli were not significant $[\mathrm{Fs}(1,10) \leqslant 2.90, \mathrm{p}>.05]$. The significant Groups by Stimuli interaction was largely attributable to the reactions of the subjects to the novel stimulus: the two groups differed reliably with respect to time spent near the novel stimulus $[t(10)=$ $3.76, \mathrm{p}<.05]$ but not with respect to time spent near the original stimulus $[\mathrm{t}(10)=1.02, \mathrm{p}>.05]$.

The reliability of the preferences exhibited by individual subjects was assessed via $t$ tests. For each subject, the scores used in the analysis represented the time spent near the novel vs. the familiar stimulus during each of the 10 stimulus cycles of the test session. In the noncontingent group, the data for three subjects yielded significant preferences, and these were for the novel stimulus $[\operatorname{ts}(9) \geqslant 4.39, \mathrm{p}<$ $.05]$. In the response-contingent group, on the other hand, only one subject exhibited a significant preference, and this was for the original imprinting stimulus $[\mathrm{t}(9)=3.99, \mathrm{p}<.05]$.

Observation of subjects during the test session via closed-circuit television revealed that during the stimulus presentation periods, after the subject had entered the quadrant closest to one or the other stimulus, the subject sometimes sat quietly in front of that stimulus and sometimes followed it along the screen. During the stimulus withdrawal periods, the subjects usually emitted distress calls and ran about the chamber in a haphazard, agitated manner. When the stimuli were again presented, all signs of agitation usually stopped immediately, and the subjects would again begin to approach one or the other stimulus. These are the typical reactions of ducklings to the presence and absence of stimuli to which they have been imprinted (Hess, 1973; Hoffman, 1968).

\section{DISCUSSION}

Previous studies by Barrett (1972) and Ratner (1976) have shown that ducklings exposed to shock in the presence of one imprinting stimulus will tend to approach and stay near a novel imprinting stimulus when both stimuli are simultaneously presented. This effect was also obtained in the present study. Subjects for whom shocks were independent of responses reliably preferred the novel imprinting stimulus. More importantly, though, the present work revealed that when subjects were shocked only when they pecked at food they failed to exhibit this effect.

These results parallel the findings of Orme-Johnson and Yarczower (1974). In their experiment, pigeons were shocked in the presence of an exteroceptive stimulus (a key light). For some of the birds shock was contingent upon a keypeck response, and for other subjects shock was response-independent. In a subsequent test, shock was discontinued and brief presentation of the stimulus was made contingent on keypecking for all subjects in order to assess the aversiveness of the stimulus. Keypecking was reduced in the response-independent subjects but not in the response-contingent birds, indicating that the stimulus had acquired conditioned aversive properties only for the birds given response-independent shocks. In the present study as well, an associative effect of shock on subjects' reactions to an exteroceptive stimulus (in this case, an imprinting stimulus) was obtained when shock was response-independent but not when shock was response-contingent. Thus, when shock delivery was response-contingent, the original imprinting stimulus acquired little or no aversiveness despite the fact that shock was repeatedly delivered in its presence.

Why should a shock-associated stimulus fail to acquire conditioned aversive properties when shock delivery is response contingent? To account for their results, Orme-Johnson and Yarczower (1974) suggested that "classical conditioning involving responseproduced stimuli may have interfered with classical conditioning involving the exteroceptive stimulus." Such an interpretation is supported by studies which demonstrate that when an animal is presented with an array of stimuli which differentially predict reinforcement it will come under the control of those stimuli which are the most reliable predictors of reinforcement (e.g., Rescorla, 1972; Wagner, Logan, Haberlandt, \& Price, 1968). For the animals given response-contingent shock in both the Orme-Johnson and Yarczower study and in the present study, response-generated cues were more reliable predictors of shock than the exteroceptive stimulus, because response-produced cues only occurred immediately before each shock, whereas the exteroceptive stimulus was continuously present between shocks. On the other hand, for animals in the response-independent groups of both studies, the exteroceptive stimulus could acquire aversive properties because there were no other stimuli present that more reliably predicted the shock. In less formal terms, we are suggesting that when an aversive event consistently follows a given response, the subject associates the event with the response rather than with the exteroceptive stimuli present in the situation. The fact that food-pecking in the present experiment was reliably suppressed by response-contingent shock is consistent with this interpretation.

In addition to its implications for the study of aversive control, the present findings are relevant to the study of attachment phenomena. Barrett's (1972) and Ratner's (1976) findings implied that the occurrence of noxious events in the presence of a social object reduces the strength of the subject's attach- 
ment to that object. The present results reveal that this reduction need not occur, provided that the noxious events are contingent upon a given response.

\section{REFERENCES}

Azrin, H. H., Ulrich, R. E., Hutchinson, R. R., \& Norman, D. G. Effect of shock duration on shock induced fighting Journal of the Experimental Analysis of Behavior, 1964, 7, 9-11. Barfield, R. J., \& SAchs, B. D. Sexual behavior: Stimulation by painful electric shock to skin in male rats. Science. 1968, 161, 392-393.

BARRETT, J. E. Schedules of electric shock presentation in the behavioral control of imprinted ducklings. Journal of the Experimental Analysis of Behavior, 1972, 18, 305-321.

Bow LBY, J. Attachment and loss (Vol. 1) Attachment. New York: Basic Books, 1969.

CAgguila, A. R., \& EIbERgen, R. Copulation of virgin male rats evoked by painful peripheral stimulation. Journal of Comparative and Physiological Psychology, 1969, 69, 414-419.

HaRLOW, H. F., \& HARLOW, M. K. Social deprivation in monkeys. Scientific American, 1962, 207, 136-146.

HEss, E. H. Imprinting: Early experience and the developmental psychology of attachment. Van Nostrand Reinhold, 1973.
Hoffman, H. S. The control of distress vocalization by an imprinted stimulus. Behaviour, 1968, 30, 175-191.

Hoffman, H. S., \& Ratner, A. M. A shock-delivery system for newly hatched precocial birds. Journal of the Experimental Analysis of Behavior, 1974, 22, 575-576.

Kovach, J. K., \& Hess, E. H. Imprinting: Effects of painful stimulation upon the following behavior. Journal of Comparative and Physiological Psychology, 1963, 56, 461-464.

O'Kelley, L. I., \& STECKLE, L. C. A note on long enduring emotional responses in the rat. Journal of Psychology, 1939, 8, 125-131.

ORME-Johnson, D. W., \& Yarczower, M. Conditioned suppression, punishment, and aversion. Joumal of the Experimental Analysis of Behavior, 1974, 21, 57-74.

RatNer, A. M. Modification of ducklings' filial behavior by aversive stimulation. Journal of Experimental Psychology: Animal Behavior Processes, 1976, 2, 266-284.

Rescorla, R. A. Informational variables in Pavlovian conditioning. In G. H. Bower (Ed.), The psychology of learning and motivation: Advances in research and theory (Vol. 6). New York: Academic Press, 1972. Pp. 1-46.

Wagner, A. R,, Logan, F. A., Haberlandt, K., \& Price, T. Stimulus selection in animal discrimination learning. Journal of Experimental Psychology, 1968, 76, 171-180.

(Received for publication May 18, 1978; accepted May 30, 1978.) 